\title{
Multilayer Laue Lenses (MLL) with 45 mm Focal Length as Optics for In-situ Nanoindentation Experiments
}

Adam Kubec ${ }^{1}$, Sven Niese ${ }^{2}$, Jürgen Gluch $^{3}$, Martin Rosenthal $^{4}$, Juraj Todt ${ }^{5}$, Jozef Keckes ${ }^{5}$ and Peter Gawlitza ${ }^{1, *}$

1. Fraunhofer IWS Dresden, EUV- and X-ray Optics, Dresden, Germany

2. AXO Dresden GmbH, Dresden, Germany

3. Fraunhofer IKTS Dresden, Dresden, Germany

4. ESRF, Microfocus Beamline ID13, Grenoble, France

5. Erich Schmid Institute for Materials Science, Leoben, Austria

* peter.gawlitza@iws.fraunhofer.de

In the past decade research activities on Multilayer Laue Lenses (MLLs) have shown encouraging results in hard X-ray microscopy. Calculations have shown their potential to reach diffraction limited resolutions in the order of $1 \mathrm{~nm}[1,2]$.

The increasing demand of in-situ and operando experiments require working distances in X-ray microscopes of at least several millimeters and up to several centimeters. This space becomes necessary to contain the experimental setup, stages and chambers as well as the samples. We have developed a monolithic 2D focusing MLL optics based on two crossed MLLs with low stress multilayers of the $\mathrm{Mo} / \mathrm{C} / \mathrm{Si} / \mathrm{C}$ system $[3,4]$. This multilayer configuration allows to deposit multilayer stacks with a total thicknesses of up to $100 \mu \mathrm{m}$. With this large aperture we designed an MLL with an extraordinary long focal length and a corresponding long working distance of several centimeters and diffraction limited focal spot sizes well below $100 \mathrm{~nm}$. A measurement with an MLL based on this multilayer system has demonstrated the capability to realize high diffraction efficiencies close to theoretical predictions [5].

The design of the long focal length MLLs is based on the Fresnel zone plate law and contains zones 4000 to 12500 with a focal length of $45 \mathrm{~mm}$ at an X-ray energy of $12 \mathrm{keV}$ with equivalent zone widths between $9.6 \mathrm{~nm}$ and $17 \mathrm{~nm}$. This results in a total thickness of slightly more than $100 \mu \mathrm{m}$. Figure 1 shows a cross section SEM image of a FIB (focused ion beam) milled lamella. Working distance, i.e. space between the order sorting pinhole and the focus position, is for this type of MLL in the order of $25 \mathrm{~mm}$ at an X-ray energy of $12 \mathrm{keV}$. The diffraction limited focal spot size of this design is in the order of $50 \mathrm{~nm}$, which is sufficient for many experimental setups and techniques. First focusing measurements with lenses based on this design have been successfully performed at the ESRF beamline ID13. The mounting of the monolithic crossed MLL housing, the pinhole and the sample is shown in Figure 2 in their respective positions as they were used for the measurement.

This ESRF ID13 setup was also used to perform in-situ indentation measurements on $15 \mu \mathrm{m}$ CrN thin film on $\mathrm{Si}(100)$ substrate, which was loaded stepwise using a wedge diamond indenter [6]. At each indentation step, WAXS frames were collected using an Eiger 4M detector. The data were used to evaluate multiaxial stress fields in the loaded film as a function of the applied load. In Figure 3a, a scanning electron micrograph from the film cross-section showing the indenter imprint with a few crosssectional cracks is presented. In Figure 3b, a cross-sectional distribution of in-plane stresses evaluated for the indenter load of $1 \mathrm{~N}$ can be seen. The resolution was limited by the roughness of the interfaces as well as the temporary setup in the first iteration of these measurements. The results indicate very complex variation of compressive intrinsic stresses with a maximum in the film center, caused by the 
variation of process parameters during the film deposition. Due to the indentation, additional anisotropic stress is initiated into the film, whose form and magnitude correlate with the cracks shown in Figure 3 a. The in-situ approach allows to reveal microstructure origins of the indentation-induced cracks in the film and to determine the magnitude of stress responsible for particular crack patterns.

References/Acknowledgement:

[1] J Maser et al, Proc. of SPIE 5539 (2004), p. 185.

[2] H Yan et al, Phys. Rev. B 76 (2007), p. 115438.

[3] A Kubec et al, J. Synchrotron Radiat. 21 (2014), p. 1122.

[4] S Niese et al, Opt. Express 22.17 (2014): 20008-20013.

[5] A Kubec et al, Appl. Phys. Lett. 110(11) (2017), p. 111905.

[6] A Zeilinger et al. Sci. Rep. 6 (2016): 22670

The authors acknowledge scientists and staff of beamline ID13 at the European Synchrotron Radiation Facility (ESRF). This work was performed in the scope of the Long Term Project MI-1216.

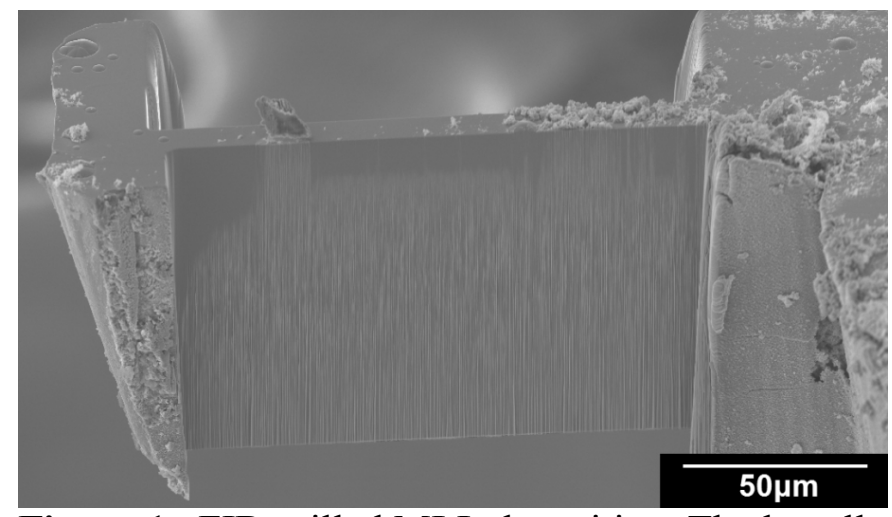

Figure 1. FIB-milled MLL deposition. The lamella is tilted by 37 degrees to the line of sight.

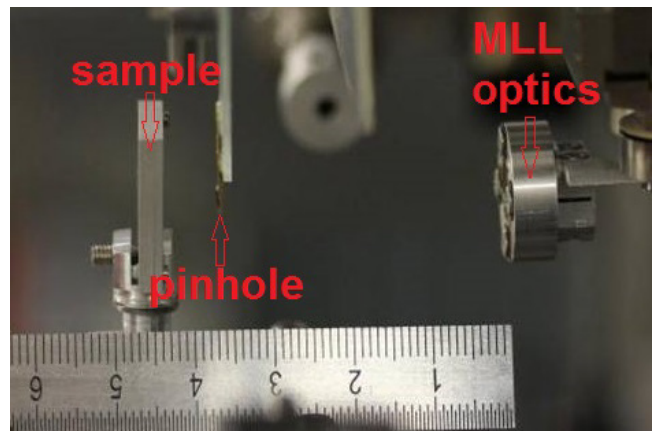

Figure 2. Photograph of the MLL setup with sample in focal plane and an absolute scale. The working distance was limited by the small opening of the available pinhole (diameter approx. $20 \mu \mathrm{m}$ ).
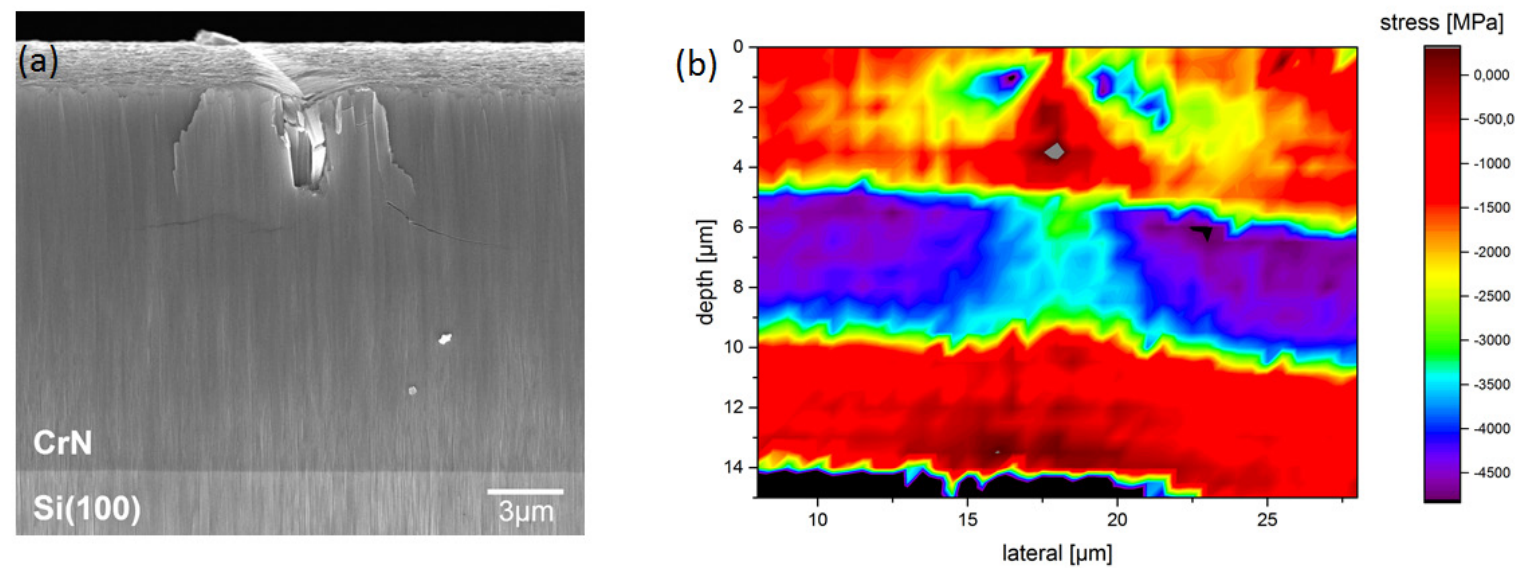

Figure 3. SEM cross-section image of a $\mathrm{CrN}$ thin film on $\mathrm{Si}(100)$ substrate with cracks formed due to in-situ indentation (a). Cross-section distribution of in-plane stress evaluated from X-ray nanodiffaction data (b) 